\title{
Performance Analysis and Coherent Guaranteed Cost Control for Uncertain Quantum Systems Using Small Gain and Popov Methods
}

\author{
Chengdi Xiang, Ian R. Petersen and Daoyi Dong
}

\begin{abstract}
This paper extends applications of the quantum small gain and Popov methods from existing results on robust stability to performance analysis results for a class of uncertain quantum systems. This class of systems involves a nominal linear quantum system and is subject to quadratic perturbations in the system Hamiltonian. Based on these two methods, coherent guaranteed cost controllers are designed for a given quantum system to achieve improved control performance. An illustrative example also shows that the quantum Popov approach can obtain less conservative results than the quantum small gain approach for the same uncertain quantum system.
\end{abstract}

\section{INTRODUCTION}

Due to recent advances in quantum and nano-technology, there has been a considerable attention focusing on research in the area of quantum feedback control systems; e.g., [1][24]. In particular, robust control has been recognized as a critical issue in quantum control systems, since many quantum systems are unavoidably subject to disturbances and uncertainties in practical applications; e.g., [1], [2] and [3]. A majority of papers in the area of quantum feedback control only consider the case in which the controller is a classical system. In this case, analog and digital electronic devices may be involved and quantum measurements are required in the feedback loop. Due to the limitations imposed by quantum mechanics on the use of quantum measurement, recent research has considered the design of coherent quantum controllers to achieve improved performance. In this case, the controller itself is a quantum system; e.g., [1], [4] and [5]. In the linear case, the quantum system is often described by linear quantum stochastic differential equations (QSDEs) that require physical realizability conditions in terms of constraints on the system matrices to represent physically meaningful systems.

As opposed to using QSDEs, the papers [6], [7] have introduced a set of parameterizations $(S, L, H)$ to represent a class of open quantum systems, where $S$ is a scattering matrix, $L$ is a vector of coupling operators and $H$ is a Hamiltonian operator. The matrix $S$, together with the vector $L$, describes the interface between the system and the field, and the operator $H$ represents the energy of the system. The advantage of using a triple $(S, L, H)$ is that this framework automatically represents a physically realizable quantum system. Therefore, in this paper, a coherent guaranteed cost

Chengdi Xiang, Ian R. Petersen and Daoyi Dong are with the School of Engineering and Information Technology, University of New South Wales at the Australian Defence Force Academy, Canberra ACT 2600, Australia. \{elyssaxiang, i.r.petersen, daoyidong\}@gmail. com This work was supported by the Australian Research Council. controller is designed based on $(S, L, H)$ and the physical realizability condition does not need to be considered.

The small gain theorem and the Popov approach are two of the most important methods for the analysis of robust stability in classical control. The paper [8] has applied the small gain method to obtain a robust stability result for uncertain quantum systems. This result gives a sufficient condition for robust stability in terms of a strict bounded real condition. The small gain method has also been extended to the robust stability analysis of quantum systems with different perturbations and applications (e.g., see [9], [10], [11] and [12]). The paper [13] has introduced a quantum version of the Popov stability criterion in terms of a frequency domain condition which is of the same form as the classical Popov stability condition (e.g., see [25]). The Popov approach has also been used to analyze the robust stability of an optical cavity containing a saturated Kerr medium [14]. Also, the paper [13] has shown that the frequency domain condition obtained in [13] is less conservative than the stability result using the small gain theorem [8].

In this paper, we extend the quantum small gain method in [8] and the Popov type approach in [13] from robust stability analysis to robust performance analysis for uncertain quantum systems. We assume that the system Hamiltonian $H$ can be decomposed in terms of a known nominal Hamiltonian $H_{1}$ and a perturbation Hamiltonian $H_{2}$, i.e., $H=$ $H_{1}+H_{2}$. The perturbation Hamiltonian $H_{2}$ is contained in a set of Hamiltonians $\mathcal{W}$. We consider uncertain quantum systems where the nominal system is a linear system and the perturbation Hamiltonian is quadratic. Moreover, a coherent controller is designed using the small gain approach and the Popov approach for the uncertain quantum system, where a guaranteed bound on a cost function is derived in terms of linear matrix inequality (LMI) conditions. Although preliminary versions of the results in this paper have been presented in the conference papers [22] and [23], this paper presents complete proofs of the main results and modifies the example in [22] for a consistent performance comparison of the proposed two methods.

The remainder of the paper proceeds as follows. In Section II. we define the general class of quantum systems under consideration and specify the nominal system as a linear quantum system. We then present a set of quadratic Hamiltonian perturbations in Section III. In Section IV] a performance cost function for the given system is defined. Moreover, a small gain approach and a Popov type method are used to analyze the performance of the given system. In Section $\mathrm{V}$, a quantum controller is added to the original 
system to stabilize the system and also to achieve improved performance. Also, corresponding approaches are used to construct a coherent guaranteed cost controller in terms of LMI conditions. In Section VI an illustrative example is provided to demonstrate the method that is developed in this paper. A performance comparison between these two methods is also shown in the illustrative example. We present some conclusions in Section VII.

\section{QUANTUM SYSTEMS}

In this section, we describe the general class of quantum systems under consideration, which is defined by parameters $(S, L, H)$. Here $H=H_{1}+H_{2}, H_{1}$ is a known self-adjoint operator on the underlying Hilbert space referred to as the nominal Hamiltonian and $\mathrm{H}_{2}$ is a self-adjoint operator on the underlying Hilbert space referred to as the perturbation Hamiltonian contained in a specified set of Hamiltonians $\mathcal{W}$; e.g., [6], [7]. The set $\mathcal{W}$ can correspond to a set of exosystems (see, [6]). The corresponding generator for this class of quantum systems is given by

$$
\mathcal{G}(X)=-i[X, H]+\mathcal{L}(X)
$$

where $\mathcal{L}(X)=\frac{1}{2} L^{\dagger}[X, L]+\frac{1}{2}\left[L^{\dagger}, X\right] L$. Here, $[X, H]=$ $X H-H X$ describes the commutator between two operators and the notation ${ }^{\dagger}$ refers to the adjoint transpose of a vector of operators. Based on a quantum stochastic differential equation, the triple $(S, L, H)$, together with the corresponding generators, defines the Heisenberg evolution $X(t)$ of an operator $X$ [6]. The results presented in this paper will build on the following results from [13].

Lemma 1: [13] Consider an open quantum system defined by $(S, L, H)$ and suppose there exist non-negative selfadjoint operators $V$ and $W$ on the underlying Hilbert space such that

$$
\mathcal{G}(V)+W \leq \lambda
$$

where $\lambda$ is a real number. Then for any plant state, we have

$$
\limsup _{T \rightarrow \infty} \frac{1}{T} \int_{0}^{T}\langle W(t)\rangle d t \leq \lambda .
$$

Here $W(t)$ denotes the Heisenberg evolution of the operator $W$ and $\langle\cdot\rangle$ denotes quantum expectation; e.g., see [13] and [6].

In this paper, the nominal system is considered to be a linear quantum system. We assume that $H_{1}$ is in the following form

$$
H_{1}=\frac{1}{2}\left[\begin{array}{ll}
a^{\dagger} & a^{T}
\end{array}\right] M\left[\begin{array}{l}
a \\
a^{\#}
\end{array}\right]
$$

where $M \in \mathbb{C}^{2 n \times 2 n}$ is a Hermitian matrix and has the following form with $M_{1}=M_{1}^{\dagger}$ and $M_{2}=M_{2}^{T}$

$$
M=\left[\begin{array}{cc}
M_{1} & M_{2} \\
M_{2}^{\#} & M_{1}^{\#}
\end{array}\right]
$$

Here $a$ is a vector of annihilation operators on the underlying Hilbert space and $a^{\#}$ is the corresponding vector of creation operators. In the case of matrices, the notation ${ }^{\dagger}$ refers to the complex conjugate transpose of a matrix. In the case of vectors of operators, the notation \# refers to the vector of adjoint operators and in the case of complex matrices, this notation refers to the complex conjugate matrix. The canonical commutation relations between annihilation and creation operators are described in the following way

$$
\begin{aligned}
{\left[\left[\begin{array}{l}
a \\
a^{\#}
\end{array}\right],\left[\begin{array}{l}
a \\
a^{\#}
\end{array}\right]^{\dagger}\right]=} & {\left[\begin{array}{l}
a \\
a^{\#}
\end{array}\right]\left[\begin{array}{l}
a \\
a^{\#}
\end{array}\right]^{\dagger} } \\
& -\left(\left[\begin{array}{l}
a \\
a^{\#}
\end{array}\right]^{\#}\left[\begin{array}{l}
a \\
a^{\#}
\end{array}\right]^{T}\right)^{T} \\
= & J
\end{aligned}
$$

where $J=\left[\begin{array}{rr}I & 0 \\ 0 & -I\end{array}\right][2]$.

The coupling vector $L$ is assumed to be of the form

$$
L=\left[\begin{array}{ll}
N_{1} & N_{2}
\end{array}\right]\left[\begin{array}{l}
a \\
a^{\#}
\end{array}\right]=\tilde{N}\left[\begin{array}{l}
a \\
a^{\#}
\end{array}\right]
$$

where $N_{1} \in \mathbb{C}^{m \times n}$ and $N_{2} \in \mathbb{C}^{m \times n}$. We also write

$$
\left[\begin{array}{l}
L \\
L^{\#}
\end{array}\right]=N\left[\begin{array}{l}
a \\
a^{\#}
\end{array}\right]=\left[\begin{array}{cc}
N_{1} & N_{2} \\
N_{2}^{\#} & N_{1}^{\#}
\end{array}\right]\left[\begin{array}{l}
a \\
a^{\#}
\end{array}\right]
$$

When the nominal Hamiltonian $H$ is a quadratic function of the creation and annihilation operators as shown in (4) and the coupling operator vector is a linear function of the creation and annihilation operators, the nominal system corresponds to a linear quantum system (see, [2]).

We consider self-adjoint "Lyapunov" operators $V$ of the form

$$
V=\left[\begin{array}{ll}
a^{\dagger} & a^{T}
\end{array}\right] P\left[\begin{array}{l}
a \\
a^{\#}
\end{array}\right]
$$

where $P \in \mathbb{C}^{2 n \times 2 n}$ is a positive definite Hermitian matrix of the form

$$
P=\left[\begin{array}{cc}
P_{1} & P_{2} \\
P_{2}^{\#} & P_{1}^{\#}
\end{array}\right]
$$

We then consider a set of non-negative self-adjoint operators $\mathcal{P}$ defined as

$$
\mathcal{P}=\left\{\begin{array}{c}
V \text { of the form }[9 \text { such that } P>0 \text { is a } \\
\text { Hermitian matrix of the form } 10]
\end{array}\right\} .
$$

\section{PERTURBATIONS OF THE HAMILTONIAN}

In Section II, we introduced the nominal linear quantum system. This section defines the quadratic Hamiltonian perturbations (e.g., see [8], [18]) for the quantum system under consideration. We first define two general sets of Hamiltonians in terms of a commutator decomposition, and then present two specific sets of quadratic Hamiltonian perturbations. 


\section{A. Commutator Decomposition}

For the set of non-negative self-adjoint operators $\mathcal{P}$ and given real parameters $\gamma>0, \delta \geq 0$, a particular set of perturbation Hamiltonians $\mathcal{W}_{1}$ is defined in terms of the commutator decomposition

$$
\left[V, H_{2}\right]=\left[V, z^{\dagger}\right] w-w^{\dagger}[z, V]
$$

for $V \in \mathcal{P}$, where $w$ and $z$ are given vectors of operators. $\mathcal{W}_{1}$ is then defined in terms of sector bound condition:

$$
w^{\dagger} w \leq \frac{1}{\gamma^{2}} z^{\dagger} z+\delta
$$

We define

$$
\mathcal{W}_{1}=\left\{\begin{array}{c}
H_{2}: \exists w, z \text { such that } 13 \text { is satisfied } \\
\text { and } 12 \text { is satisfied } \forall V \in \mathcal{P}
\end{array}\right\} .
$$

\section{B. Alternative Commutator Decomposition}

Given a set of non-negative operators $\mathcal{P}$, a self-adjoint operator $H_{1}$, a coupling operator $L$, real parameters $\beta \geq 0$ $\gamma>0$, and a set of Popov scaling parameters $\Theta \subset[0, \infty)$, we define a set of perturbation Hamiltonians $\mathcal{W}_{2}$ in terms of the commutator decompositions [13]

$$
\begin{aligned}
& {\left[V-\theta H_{1}, H_{2}\right] }=\left[V-\theta H_{1}, z^{\dagger}\right] w-w^{\dagger}\left[z, V-\theta H_{1}\right] \\
& \mathcal{L}\left(H_{2}\right) \leq \mathcal{L}\left(z^{\dagger}\right) w+w^{\dagger} \mathcal{L}(z)+\beta[z, L]^{\dagger}[z, L]
\end{aligned}
$$

for $V \in \mathcal{P}$ and $\theta \in \Theta$, where $w$ and $z$ are given vectors of operators. Note that 12 and $(15)$ correspond to a general quadratic perturbation of the Hamiltonian. This set $\mathcal{W}_{2}$ is then defined in terms of the sector bound condition

$$
\left(w-\frac{1}{\gamma} z\right)^{\dagger}\left(w-\frac{1}{\gamma} z\right) \leq \frac{1}{\gamma^{2}} z^{\dagger} z .
$$

We define

$$
\mathcal{W}_{2}=\left\{\begin{array}{c}
H_{2} \geq 0: \exists w, z \text { such that } 15 \text { and } 16 \\
\text { are satisfied } \forall V \in \mathcal{P}, \theta \in \Theta
\end{array}\right\} .
$$

\section{Quadratic Hamiltonian Perturbation}

We consider a set of quadratic perturbation Hamiltonians that is in the form

$$
H_{2}=\frac{1}{2}\left[\begin{array}{ll}
\zeta^{\dagger} & \zeta^{T}
\end{array}\right] \Delta\left[\begin{array}{l}
\zeta \\
\zeta^{\#}
\end{array}\right]
$$

where $\zeta=E_{1} a+E_{2} a^{\#}$ and $\Delta \in \mathbb{C}^{2 m \times 2 m}$ is a Hermitian matrix of the form

$$
\Delta=\left[\begin{array}{cc}
\Delta_{1} & \Delta_{2} \\
\Delta_{2}^{\#} & \Delta_{1}^{\#}
\end{array}\right]
$$

with $\Delta_{1}=\Delta_{1}^{\dagger}$ and $\Delta_{2}=\Delta_{2}^{T}$.

Since the nominal system is linear, we use the relationship:

$$
z=\left[\begin{array}{l}
\zeta \\
\zeta^{\#}
\end{array}\right]=\left[\begin{array}{ll}
E_{1} & E_{2} \\
E_{2}^{\#} & E_{1}^{\#}
\end{array}\right]\left[\begin{array}{l}
a \\
a^{\#}
\end{array}\right]=E\left[\begin{array}{l}
a \\
a^{\#}
\end{array}\right]
$$

Then

$$
H_{2}=\frac{1}{2}\left[\begin{array}{ll}
a^{\dagger} & a^{T}
\end{array}\right] E^{\dagger} \Delta E\left[\begin{array}{l}
a \\
a^{\#}
\end{array}\right]
$$

When the matrix $\Delta$ is subject to the norm bound

$$
\|\Delta\| \leq \frac{2}{\gamma}
$$

where $\|$.$\| refers to the matrix induced norm, we define$

$$
\mathcal{W}_{3}=\left\{\begin{array}{c}
\left.H_{2} \text { of the form } 18\right) \text { and } 19 \text { such that } \\
\text { condition } 22 \text { is satisfied }
\end{array}\right\} \text {. }
$$

In [8], it has been proven that for any set of self-adjoint operators $\mathcal{P}$,

$$
\mathcal{W}_{3} \subset \mathcal{W}_{1}
$$

When the matrix $\Delta$ is subject to the bounds

$$
0 \leq \Delta \leq \frac{4}{\gamma} I
$$

we define

$$
\mathcal{W}_{4}=\left\{\begin{array}{c}
H_{2} \text { of the form } 18 \text { and }(19) \text { such that } \\
\text { condition } 25 \text { is satisfied }
\end{array}\right\} .
$$

In [13], it has been proven that if $[z, L]$ is a constant vector, then for any set of self-adjoint operators $\mathcal{P}$,

$$
\mathcal{W}_{4} \subset \mathcal{W}_{2}
$$

\section{PERFORMANCE ANALYSIS}

In this section, we provide several results on performance analysis for quantum systems subject to a quadratic perturbation Hamiltonian. Also, the associated cost function is defined in the following way:

$$
\mathcal{J}=\limsup _{T \rightarrow \infty} \frac{1}{T} \int_{0}^{T}\left\langle\left[\begin{array}{ll}
a^{\dagger} & a^{T}
\end{array}\right] R\left[\begin{array}{l}
a \\
a^{\#}
\end{array}\right]\right\rangle d t
$$

where $R>0$. We denote

$$
W=\left[\begin{array}{ll}
a^{\dagger} & a^{T}
\end{array}\right] R\left[\begin{array}{l}
a \\
a^{\#}
\end{array}\right] .
$$

In order to prove the following theorems on performance analysis, we require some algebraic identities.

Lemma 2: (See Lemma 4 of [8]) Suppose $V \in \mathcal{P}, H_{1}$ is of the form (4) and $L$ is of the form (7). Then

$$
\begin{gathered}
{\left[V, H_{1}\right]=\left[\begin{array}{l}
a \\
a^{\#}
\end{array}\right]^{\dagger}(P J M-M J P)\left[\begin{array}{l}
a \\
a^{\#}
\end{array}\right],} \\
\mathcal{L}(V)=-\frac{1}{2}\left[\begin{array}{l}
a \\
a^{\#}
\end{array}\right]^{\dagger}\left(N^{\dagger} J N J P+P J N^{\dagger} J N\right)\left[\begin{array}{l}
a \\
a^{\#}
\end{array}\right] \\
+\operatorname{Tr}\left(P J N^{\dagger}\left[\begin{array}{ll}
I & 0 \\
0 & 0
\end{array}\right] N J\right), \\
{\left[\left[\begin{array}{l}
a \\
a^{\#}
\end{array}\right],\left[\begin{array}{l}
a \\
a^{\#}
\end{array}\right]^{\dagger} P\left[\begin{array}{l}
a \\
a^{\#}
\end{array}\right]\right]=2 J P\left[\begin{array}{l}
a \\
a^{\#}
\end{array}\right]}
\end{gathered}
$$

Lemma 3: For $V \in \mathcal{P}$ and $z$ defined in (20),

$$
[z, V]=2 E J P\left[\begin{array}{l}
a \\
a^{\#}
\end{array}\right],
$$

$$
\left[V, z^{\dagger}\right][z, V]=4\left[\begin{array}{l}
a \\
a^{\#}
\end{array}\right]^{\dagger} P J E^{\dagger} E J P\left[\begin{array}{l}
a \\
a^{\#}
\end{array}\right]
$$




$$
z^{\dagger} z=\left[\begin{array}{l}
a \\
a^{\#}
\end{array}\right]^{\dagger} E^{\dagger} E\left[\begin{array}{l}
a \\
a^{\#}
\end{array}\right] .
$$

Proof: The proof follows from Lemma 2 .

Lemma 4: (See Lemma 5 of [13]) For $z$ defined in 20) and $L$ being of the form (7),

$$
[z, L]=\left[E\left[\begin{array}{l}
a \\
a^{\#}
\end{array}\right], \tilde{N}\left[\begin{array}{l}
a \\
a^{\#}
\end{array}\right]\right]=E J \Sigma \tilde{N}^{T}
$$

is a constant vector, where

$$
\Sigma=\left[\begin{array}{ll}
0 & I \\
I & 0
\end{array}\right] .
$$

Lemma 5: (See Lemma 6 of [13]) For $z$ defined in 20], $H_{1}$ defined in (4) and $L$ being of the form (7), we have

$$
-i\left[z, H_{1}\right]+\mathcal{L}(z)=E\left(-i J M-\frac{1}{2} J N^{\dagger} J N\right)\left[\begin{array}{l}
a \\
a^{\#}
\end{array}\right]
$$

and

$$
i[z, V]=2 i E J P\left[\begin{array}{l}
a \\
a^{\#}
\end{array}\right] .
$$

Now we present two theorems (Theorem 1 and Theorem 2) which can be used to analyze the performance of the given quantum systems using a quantum small gain method and a Popov type approach, respectively.

\section{A. Performance Analysis Using the Small Gain Approach}

Theorem 1: Consider an uncertain quantum system ( $S, L, H)$, where $H=H_{1}+H_{2}, H_{1}$ is in the form of (4), $L$ is of the form 77 and $H_{2} \in \mathcal{W}_{3}$. If $F=-i J M-\frac{1}{2} J N^{\dagger} J N$ is Hurwitz,

$$
\left[\begin{array}{cc}
F^{\dagger} P+P F+\frac{E^{\dagger} E}{\gamma^{2} \tau^{2}}+R & 2 P J E^{\dagger} \\
2 E J P & -I / \tau^{2}
\end{array}\right]<0
$$

has a solution $P>0$ in the form of 10$]$ and $\tau>0$, then

$$
\begin{aligned}
\mathcal{J} & =\limsup _{T \rightarrow \infty} \frac{1}{T} \int_{0}^{T}\langle W(t)\rangle d t \\
& =\limsup _{T \rightarrow \infty} \frac{1}{T} \int_{0}^{T}\left\langle\left[\begin{array}{ll}
a^{\dagger} & a^{T}
\end{array}\right] R\left[\begin{array}{l}
a \\
a^{\#}
\end{array}\right]\right\rangle d t \leq \tilde{\lambda}+\frac{\delta}{\tau^{2}}
\end{aligned}
$$

where

$$
\tilde{\lambda}=\operatorname{Tr}\left(P J N^{\dagger}\left[\begin{array}{ll}
I & 0 \\
0 & 0
\end{array}\right] N J\right) .
$$

In order to prove this theorem, we need the following lemma.

Lemma 6: Consider an open quantum system $(S, L, H)$ where $H=H_{1}+H_{2}$ and $H_{2} \in \mathcal{W}_{1}$, and the set of nonnegative self-adjoint operators $\mathcal{P}$. If there exists a $V \in \mathcal{P}$ and real constants $\tilde{\lambda} \geq 0, \tau>0$ such that

$$
-i\left[V, H_{1}\right]+\mathcal{L}(V)+\tau^{2}\left[V, z^{\dagger}\right][z, V]+\frac{1}{\gamma^{2} \tau^{2}} z^{\dagger} z+W \leq \tilde{\lambda},
$$

then

$$
\limsup _{T \rightarrow \infty} \frac{1}{T} \int_{0}^{T}\langle W(t)\rangle d t \leq \tilde{\lambda}+\frac{\delta}{\tau^{2}}, \forall t \geq 0
$$

Proof: Since $V \in \mathcal{P}$ and $H_{2} \in \mathcal{W}_{1}$,

$$
\mathcal{G}(V)=-i\left[V, H_{1}\right]+\mathcal{L}(V)-i\left[V, z^{\dagger}\right] w+i w^{\dagger}[z, V] .
$$

Also,

$$
\begin{aligned}
0 & \leq\left(\tau\left[V, z^{\dagger}\right]-\frac{i}{\tau} w^{\dagger}\right)\left(\tau\left[V, z^{\dagger}\right]-\frac{i}{\tau} w^{\dagger}\right)^{\dagger} \\
& =\tau^{2}\left[V, z^{\dagger}\right][z, V]+i\left[V, z^{\dagger}\right] w-i w^{\dagger}[z, V]+\frac{w^{\dagger} w}{\tau^{2}} .
\end{aligned}
$$

Substituting (45) into (46) and using the sector bound condition $[13$, the following inequality is obtained:

$\mathcal{G}(V) \leq-i\left[V, H_{1}\right]+\mathcal{L}(V)+\tau^{2}\left[V, z^{\dagger}\right][z, V]+\frac{1}{\gamma^{2} \tau^{2}} z^{\dagger} z+\frac{\delta}{\tau^{2}}$.

Hence,

$$
\mathcal{G}(V)+W \leq \tilde{\lambda}+\frac{\delta}{\tau^{2}} .
$$

Consequently, the conclusion in the lemma follows from Lemma 1

Proof of Theorem 17. Using the Schur complement [26], the inequality (40) is equivalent to

$$
F^{\dagger} P+P F+4 \tau^{2} P J E^{\dagger} E J P+\frac{E^{\dagger} E}{\gamma^{2} \tau^{2}}+R<0 .
$$

If the Riccati inequality (49) has a solution $P>0$ of the form (10) and $\tau>0$, according to Lemma 2 and Lemma 3 . we have

$$
\begin{aligned}
& -i\left[V, H_{1}\right]+\mathcal{L}(V)+\tau^{2}\left[V, z^{\dagger}\right][z, V]+\frac{1}{\gamma^{2} \tau^{2}} z^{\dagger} z+W= \\
& {\left[\begin{array}{l}
a \\
a^{\#}
\end{array}\right]^{\dagger}\left(\begin{array}{l}
F^{\dagger} P+P F+4 \tau^{2} P J E^{\dagger} E J P \\
+\frac{E^{\dagger} E}{\gamma^{2} \tau^{2}}+R
\end{array}\right)\left[\begin{array}{l}
a \\
a^{\#}
\end{array}\right]} \\
& \quad+\operatorname{Tr}\left(P J N^{\dagger}\left[\begin{array}{ll}
I & 0 \\
0 & 0
\end{array}\right] N J\right) .
\end{aligned}
$$

Therefore, it follows from (40) that condition (43) is satisfied with

$$
\tilde{\lambda}=\operatorname{Tr}\left(P J N^{\dagger}\left[\begin{array}{ll}
I & 0 \\
0 & 0
\end{array}\right] N J\right) \geq 0 .
$$

Then, according to the relationship 24] and Lemma 6, we have

$$
\begin{aligned}
& \limsup _{T \rightarrow \infty} \frac{1}{T} \int_{0}^{T}\langle W(t)\rangle d t \\
& =\limsup _{T \rightarrow \infty} \frac{1}{T} \int_{0}^{T}\left\langle\left[\begin{array}{ll}
a^{\dagger} & a^{T}
\end{array}\right] R\left[\begin{array}{l}
a \\
a^{\#}
\end{array}\right]\right\rangle d t \leq \tilde{\lambda}+\frac{\delta}{\tau^{2}} .
\end{aligned}
$$

\section{B. Performance Analysis Using the Popov Approach}

Theorem 2: Consider an uncertain quantum system ( $S, L, H)$, where $H=H_{1}+H_{2}, H_{1}$ is in the form of (4), $L$ is of the form 77 and $H_{2} \in \mathcal{W}_{4}$. If $F=-i J M-\frac{1}{2} J N^{\dagger} J N$ is Hurwitz, and

$$
\left[\begin{array}{cc}
P F+F^{\dagger} P+R & -2 i P J E^{\dagger}+E^{\dagger}+\theta F^{\dagger} E^{\dagger} \\
2 i E J P+E+\theta E F & -\gamma I
\end{array}\right]<0
$$


has a solution $P>0$ in the form of 10 for some $\theta \geq 0$, then

$$
\begin{aligned}
\mathcal{J} & =\limsup _{T \rightarrow \infty} \frac{1}{T} \int_{0}^{T}\langle W(t)\rangle d t \\
& =\limsup _{T \rightarrow \infty} \frac{1}{T} \int_{0}^{T}\left\langle\left[\begin{array}{ll}
a^{\dagger} & a^{T}
\end{array}\right] R\left[\begin{array}{l}
a \\
a^{\#}
\end{array}\right]\right\rangle d t \leq \lambda
\end{aligned}
$$

where

$$
\lambda=\operatorname{Tr}\left(P J N^{\dagger}\left[\begin{array}{ll}
I & 0 \\
0 & 0
\end{array}\right] N J\right)+\frac{4 \theta}{\gamma} \tilde{N}^{\#} \Sigma J E^{\dagger} E J \Sigma \tilde{N}^{T} .
$$

In order to prove this theorem, we need the following lemma.

Lemma 7: (See Theorem 1 of [13]) Consider a set of nonnegative self-adjoint operators $\mathcal{P}$, an open quantum system $(S, L, H)$ and an observable $W$, where $H=H_{1}+H_{2}$ and $H_{2} \in \mathcal{W}_{2}$ defined in (17). Suppose there exists a $V \in \mathcal{P}$ and real constants $\theta \in \Theta, \lambda \geq 0$ such that

$$
\begin{aligned}
& -i\left[V, H_{1}\right]+\mathcal{L}(V)+\frac{1}{\gamma}\left(i\left[z, V-\theta H_{1}\right]+\theta \mathcal{L}(z)+z\right)^{\dagger} \\
& \times\left(i\left[z, V-\theta H_{1}\right]+\theta \mathcal{L}(z)+z\right)+\theta \beta[z, L]^{\dagger}[z, L]+W \leq \lambda .
\end{aligned}
$$

Then

$$
\limsup _{T \rightarrow \infty} \frac{1}{T} \int_{0}^{T}\langle W(t)\rangle d t \leq \lambda .
$$

Here $W(t)$ denotes the Heisenberg evolution of the operator $W$ and $\langle\cdot\rangle$ denotes quantum expectation.

Proof of Theorem 2. Using the Schur complement, (53) is equivalent to

$$
\begin{aligned}
& P F+F^{\dagger} P+\frac{1}{\gamma}\left(-2 i P J E^{\dagger}+E^{\dagger}+\theta F^{\dagger} E^{\dagger}\right) \\
& \times(2 i E J P+E+\theta E F)+R<0 .
\end{aligned}
$$

According to Lemma 2 and Lemma 5, we have

$$
\begin{aligned}
& -i\left[V, H_{1}\right]+\mathcal{L}(V)+\frac{1}{\gamma}\left(i\left[z, V-\theta H_{1}\right]+\theta \mathcal{L}(z)+z\right)^{\dagger} \\
& \times\left(i\left[z, V-\theta H_{1}\right]+\theta \mathcal{L}(z)+z\right)+\frac{4 \theta}{\gamma}[z, L]^{\dagger}[z, L]+W \\
& =\left[\begin{array}{l}
a \\
a^{\#}
\end{array}\right]^{\dagger}\left(\begin{array}{c}
P F+F^{\dagger} P \\
+\frac{1}{\gamma}\left(-2 i P J E^{\dagger}+E^{\dagger}+\theta F^{\dagger} E^{\dagger}\right) \\
\times(2 i E J P+E+\theta E F)+R
\end{array}\right)\left[\begin{array}{l}
a \\
a^{\#}
\end{array}\right] \\
& +\operatorname{Tr}\left(P J N^{\dagger}\left[\begin{array}{cc}
I & 0 \\
0 & 0
\end{array}\right] N J\right)+\frac{4 \theta}{\gamma} \tilde{N}^{\#} \Sigma J E^{\dagger} E J \Sigma \tilde{N}^{T} .
\end{aligned}
$$

From this and using the relationship 27, Lemma 4 and Lemma 7, we obtain

$$
\begin{aligned}
& \limsup _{T \rightarrow \infty} \frac{1}{T} \int_{0}^{T}\langle W(t)\rangle d t \\
& =\limsup _{T \rightarrow \infty} \frac{1}{T} \int_{0}^{\infty}\left\langle\left[\begin{array}{l}
a \\
a^{\#}
\end{array}\right]^{\dagger} R\left[\begin{array}{l}
a \\
a^{\#}
\end{array}\right]\right\rangle d t \\
& \leq \lambda
\end{aligned}
$$

where

$$
\lambda=\operatorname{Tr}\left(P J N^{\dagger}\left[\begin{array}{ll}
I & 0 \\
0 & 0
\end{array}\right] N J\right)+\frac{4 \theta}{\gamma} \tilde{N}^{\#} \Sigma J E^{\dagger} E J \Sigma \tilde{N}^{T} .
$$

\section{COHERENT GUARANTEED COST CONTROLLER DESIGN}

In this section, we design a coherent guaranteed cost controller for the uncertain quantum system subject to a quadratic perturbation Hamiltonian to make the control system not only stable but also to achieve an adequate level of performance. The coherent controller is realized by adding a controller Hamiltonian $\mathrm{H}_{3} . \mathrm{H}_{3}$ is assumed to be in the form

$$
H_{3}=\frac{1}{2}\left[\begin{array}{ll}
a^{\dagger} & a^{T}
\end{array}\right] K\left[\begin{array}{l}
a \\
a^{\#}
\end{array}\right]
$$

where $K \in \mathbb{C}^{2 n \times 2 n}$ is a Hermitian matrix of the form

$$
K=\left[\begin{array}{cc}
K_{1} & K_{2} \\
K_{2}^{\#} & K_{1}^{\#}
\end{array}\right]
$$

and $K_{1}=K_{1}^{\dagger}, K_{2}=K_{2}^{T}$. Associated with this system is the cost function $\mathcal{J}$

$$
\mathcal{J}=\limsup _{T \rightarrow \infty} \frac{1}{T} \int_{0}^{\infty}\left\langle\left[\begin{array}{l}
a \\
a^{\#}
\end{array}\right]^{\dagger}\left(R+\rho K^{2}\right)\left[\begin{array}{l}
a \\
a^{\#}
\end{array}\right]\right\rangle d t
$$

where $\rho \in(0, \infty)$ is a weighting factor. We let

$$
W=\left[\begin{array}{l}
a \\
a^{\#}
\end{array}\right]^{\dagger}\left(R+\rho K^{2}\right)\left[\begin{array}{l}
a \\
a^{\#}
\end{array}\right] \text {. }
$$

The following theorems (Theorem 3 and Theorem 4) present our main results on coherent guaranteed cost controller design for the given quantum system using a quantum small gain method and a Popov type approach, respectively.

\section{A. Coherent Controller Design Using the Small Gain Ap- proach}

Theorem 3: Consider an uncertain quantum system $(S, L, H)$, where $H=H_{1}+H_{2}+H_{3}, H_{1}$ is in the form of (4), $L$ is of the form (7), $H_{2} \in \mathcal{W}_{3}$ and the controller Hamiltonian $H_{3}$ is in the form of 62. With $Q=P^{-1}$, $Y=K Q$ and $F=-i J M-\frac{1}{2} J N^{\dagger} J N$, if there exist a matrix $Q=q * I$ ( $q$ is a constant scalar and $I$ is the identity matrix), a Hermitian matrix $Y$ and a constant $\tau>0$, such that

$$
\left[\begin{array}{cccc}
A+4 \tau^{2} J E^{\dagger} E J & Y & q R^{\frac{1}{2}} & q E^{\dagger} \\
Y & -I / \rho & 0 & 0 \\
q R^{\frac{1}{2}} & 0 & -I & 0 \\
q E & 0 & 0 & -\gamma^{2} \tau^{2} I
\end{array}\right]<0
$$

where $A=q F^{\dagger}+F q+i Y J-i J Y$, then the associated cost function satisfies the bound

$$
\begin{aligned}
& \limsup _{T \rightarrow \infty} \frac{1}{T} \int_{0}^{T}\langle W(t)\rangle d t \\
& =\limsup _{T \rightarrow \infty} \frac{1}{T} \int_{0}^{\infty}\left\langle\left[\begin{array}{l}
a \\
a^{\#}
\end{array}\right]^{\dagger}\left(R+\rho K^{2}\right)\left[\begin{array}{l}
a \\
a^{\#}
\end{array}\right]\right\rangle d t \\
& \leq \tilde{\lambda}+\frac{\delta}{\tau^{2}}
\end{aligned}
$$


where

$$
\tilde{\lambda}=\operatorname{Tr}\left(P J N^{\dagger}\left[\begin{array}{ll}
I & 0 \\
0 & 0
\end{array}\right] N J\right) .
$$

Proof: Suppose the conditions of the theorem are satisfied. Using the Schur complement, (66) is equivalent to

$$
\left[\begin{array}{ccc}
A+4 \tau^{2} J E^{\dagger} E J+\rho Y Y & q R^{\frac{1}{2}} & q E^{\dagger} \\
q R^{\frac{1}{2}} & -I & 0 \\
q E & 0 & -\gamma^{2} \tau^{2} I
\end{array}\right]<0 .
$$

Applying the Schur complement again, it follows that 69. is equivalent to

$$
\left[\begin{array}{cc}
A+4 \tau^{2} J E^{\dagger} E J+\rho Y Y+q^{2} R & q E^{\dagger} \\
q E & -\gamma^{2} \tau^{2} I
\end{array}\right]<0
$$

and 70 ) is equivalent to

$$
\begin{aligned}
& q F^{\dagger}+F q+i Y J-i J Y+4 \tau^{2} J E^{\dagger} E J \\
& +\rho Y Y+q^{2}\left(\frac{E^{\dagger} E}{\gamma^{2} \tau^{2}}+R\right)<0 .
\end{aligned}
$$

Substituting $Y=K q=q K^{\dagger}$ into 71 , we obtain

$$
\begin{aligned}
& q(F-i J K)^{\dagger}+(F-i J K) q+4 \tau^{2} J E^{\dagger} E J \\
& +q^{2}\left(\frac{E^{\dagger} E}{\gamma^{2} \tau^{2}}+R+\rho K^{2}\right)<0 .
\end{aligned}
$$

Since $P=Q^{-1}$, premultiplying and postmultiplying this inequality by the matrix $P$, we have

$$
\begin{aligned}
& (F-i J K)^{\dagger} P+P(F-i J K)+4 \tau^{2} P J E^{\dagger} E J P \\
& +\frac{E^{\dagger} E}{\gamma^{2} \tau^{2}}+R+\rho K^{2}<0 .
\end{aligned}
$$

It follows straightforwardly from (73) that $F-i J K$ is Hurwitz. We also know that

$$
\begin{aligned}
& -i\left[V, H_{1}+H_{3}\right]+\mathcal{L}(V)+\tau^{2}\left[V, z^{\dagger}\right][z, V]+\frac{1}{\gamma^{2} \tau^{2}} z^{\dagger} z+W \\
& =\left[\begin{array}{l}
a \\
a^{\#}
\end{array}\right]^{\dagger}\left(\begin{array}{l}
(F-i J K)^{\dagger} P+P(F-i J K) \\
+4 \tau^{2} P J E^{\dagger} E J P+E^{\dagger} E /\left(\gamma^{2} \tau^{2}\right) \\
+R+\rho K^{2}
\end{array}\right)\left[\begin{array}{l}
a \\
a^{\#}
\end{array}\right] \\
& +\operatorname{Tr}\left(P J N^{\dagger}\left[\begin{array}{ll}
I & 0 \\
0 & 0
\end{array}\right] N J\right) .
\end{aligned}
$$

According to the relationship (24) and Lemma 6 , we have

$$
\begin{aligned}
& \limsup _{T \rightarrow \infty} \frac{1}{T} \int_{0}^{T}\langle W(t)\rangle d t \\
& =\limsup _{T \rightarrow \infty} \frac{1}{T} \int_{0}^{\infty}\left\langle\left[\begin{array}{l}
a \\
a^{\#}
\end{array}\right]^{\dagger}\left(R+\rho K^{2}\right)\left[\begin{array}{l}
a \\
a^{\#}
\end{array}\right]\right\rangle d t \\
& \leq \tilde{\lambda}+\frac{\delta}{\tau^{2}}
\end{aligned}
$$

where

$$
\tilde{\lambda}=\operatorname{Tr}\left(P J N^{\dagger}\left[\begin{array}{ll}
I & 0 \\
0 & 0
\end{array}\right] N J\right) .
$$

Remark 1: In order to design a coherent controller which minimizes the cost bound (67) in Theorem 3, we need to formulate an inequality

$$
\operatorname{Tr}\left(P J N^{\dagger}\left[\begin{array}{ll}
I & 0 \\
0 & 0
\end{array}\right] N J\right)+\frac{\delta}{\tau^{2}} \leq \xi .
$$

We know that $P=Q^{-1}=q^{-1} I$ and apply the Schur complement to inequality (77), so that we have

$$
\left[\begin{array}{cc}
-\xi+\frac{\delta}{\tau^{2}} & B^{\frac{1}{2}} \\
B^{\frac{1}{2}} & -q
\end{array}\right] \leq 0
$$

where $B=\operatorname{Tr}\left(J N^{\dagger}\left[\begin{array}{ll}I & 0 \\ 0 & 0\end{array}\right] N J\right)$. Applying the Schur complement again, it is clear that (78) is equivalent to

$$
\left[\begin{array}{ccc}
-\xi & \delta^{\frac{1}{2}} & B^{\frac{1}{2}} \\
\delta^{\frac{1}{2}} & -\tau^{2} & 0 \\
B^{\frac{1}{2}} & 0 & -q
\end{array}\right] \leq 0
$$

Hence, we minimize $\xi$ subject to $(79)$ and $(66)$ in Theorem 3 . This is a standard LMI problem.

\section{B. Coherent Controller Design Using the Popov Approach}

Theorem 4: Consider an uncertain quantum system ( $S, L, H)$, where $H=H_{1}+H_{2}+H_{3}, H_{1}$ is in the form of (4), $L$ is of the form (7), $H_{2} \in \mathcal{W}_{4}$, the controller Hamiltonian $\mathrm{H}_{3}$ is in the form of (62). With $Q=P^{-1}$, $Y=K Q$ and $F=-i J M-\frac{1}{2} J N^{\dagger} J N$, if there exist a matrix $Q=q * I$ ( $q$ is a constant scalar and $I$ is the identity matrix), a Hermitian matrix $Y$ and a constant $\theta>0$, such that

$$
\left[\begin{array}{cccc}
A & B^{\dagger} & Y & q R^{\frac{1}{2}} \\
B & -\gamma I & 0 & 0 \\
Y & 0 & -I / \rho & 0 \\
q R^{\frac{1}{2}} & 0 & 0 & -I
\end{array}\right]<0
$$

where $A=F q+q F^{\dagger}-i J Y+i Y J$ and $B=2 i E J+E q+$ $\theta E F q-i \theta E J Y$, then the associated cost function satisfies the bound

$$
\begin{aligned}
& \limsup _{T \rightarrow \infty} \frac{1}{T} \int_{0}^{T}\langle W(t)\rangle d t \\
& =\limsup _{T \rightarrow \infty} \frac{1}{T} \int_{0}^{\infty}\left\langle\left[\begin{array}{l}
a \\
a^{\#}
\end{array}\right]^{\dagger}\left(R+\rho K^{2}\right)\left[\begin{array}{l}
a \\
a^{\#}
\end{array}\right]\right\rangle d t \leq \lambda
\end{aligned}
$$

where

$$
\lambda=\operatorname{Tr}\left(P J N^{\dagger}\left[\begin{array}{ll}
I & 0 \\
0 & 0
\end{array}\right] N J\right)+\frac{4 \theta}{\gamma} \tilde{N}^{\#} \Sigma J E^{\dagger} E J \Sigma \tilde{N}^{T} .
$$

Proof: Suppose the conditions of the theorem are satisfied. Using the Schur complement, 80 is equivalent to

$$
\left[\begin{array}{ccc}
A+\frac{1}{\gamma} B^{\dagger} B & Y & q R^{\frac{1}{2}} \\
Y & -I / \rho & 0 \\
q R^{\frac{1}{2}} & 0 & -I
\end{array}\right]<0 .
$$

We then apply the Schur complement to inequality 83 and obtain

$$
\left[\begin{array}{cc}
A+\frac{1}{\gamma} B^{\dagger} B+\rho Y Y & q R^{\frac{1}{2}} \\
q R^{\frac{1}{2}} & -I
\end{array}\right]<0 .
$$


Also, 84 is equivalent to

$$
\begin{aligned}
& F q+q F^{\dagger}-i J Y+i Y J+ \\
& \frac{1}{\gamma}\left(-2 i J E^{\dagger}+q E^{\dagger}+\theta q F^{\dagger} E^{\dagger}+i \theta Y J E^{\dagger}\right) \\
& \times(2 i E J+E q+\theta E F q-i \theta E J Y) \\
& +q^{2} R+\rho Y Y<0 .
\end{aligned}
$$

Substituting $Y=K q=q K^{\dagger}$ into 85 , we obtain

$$
\begin{aligned}
& (F-i J K) q+q(F-i J K)^{\dagger} \\
& +\frac{1}{\gamma}\left(-2 i J E^{\dagger}+q E^{\dagger}+\theta q(F-i J K)^{\dagger} E^{\dagger}\right) \\
& \times(2 i E J+E q+\theta E(F-i J K) q) \\
& +q^{2}\left(R+\rho K^{2}\right)<0 .
\end{aligned}
$$

Since $P=Q^{-1}$, premultiplying and postmultiplying this inequality by the matrix $P$, we have

$$
\begin{aligned}
& P(F-i J K)+(F-i J K)^{\dagger} P \\
& +\frac{1}{\gamma}\left(-2 i P J E^{\dagger}+E^{\dagger}+\theta(F-i J K)^{\dagger} E^{\dagger}\right) \\
& \times(2 i E J P+E+\theta E(F-i J K))+R+\rho K^{2}<0 .
\end{aligned}
$$

It follows straightforwardly from (87) that $F-i J K$ is Hurwitz. We also know that

$$
\begin{aligned}
- & i\left[V, H_{1}+H_{3}\right]+\mathcal{L}(V) \\
+ & \frac{1}{\gamma}\left(i\left[z, V-\theta\left(H_{1}+H_{3}\right)\right]+\theta \mathcal{L}(z)+z\right)^{\dagger} \\
\times & \left(i\left[z, V-\theta\left(H_{1}+H_{3}\right)\right]+\theta \mathcal{L}(z)+z\right) \\
+ & \frac{4 \theta}{\gamma}[z, L]^{\dagger}[z, L]+W \\
= & {\left[\begin{array}{l}
a \\
a^{\#}
\end{array}\right]^{\dagger} \tilde{M}\left[\begin{array}{l}
a \\
a^{\#}
\end{array}\right] } \\
& +\operatorname{Tr}\left(P J N^{\dagger}\left[\begin{array}{ll}
I & 0 \\
0 & 0
\end{array}\right] N J\right)+\frac{4 \theta}{\gamma} \tilde{N}^{\#} \Sigma J E^{\dagger} E J \Sigma \tilde{N}^{T}
\end{aligned}
$$

where

$$
\begin{aligned}
\tilde{M}= & P(F-i J K)+(F-i J K)^{\dagger} P \\
& +\frac{1}{\gamma}\left(-2 i P J E^{\dagger}+E^{\dagger}+\theta(F-i J K)^{\dagger} E^{\dagger}\right) \\
& \times(2 i E J P+E+\theta E(F-i J K)) \\
& +R+\rho K^{2} .
\end{aligned}
$$

According to the relationship 27 and Lemma 7 we have

$$
\begin{aligned}
& \limsup _{T \rightarrow \infty} \frac{1}{T} \int_{0}^{T}\langle W(t)\rangle d t \\
& =\limsup _{T \rightarrow \infty} \frac{1}{T} \int_{0}^{\infty}\left\langle\left[\begin{array}{l}
a \\
a^{\#}
\end{array}\right]^{\dagger}\left(R+\rho K^{2}\right)\left[\begin{array}{l}
a \\
a^{\#}
\end{array}\right]\right\rangle d t \\
& \leq \lambda
\end{aligned}
$$

where

$$
\lambda=\operatorname{Tr}\left(P J N^{\dagger}\left[\begin{array}{ll}
I & 0 \\
0 & 0
\end{array}\right] N J\right)+\frac{4 \theta}{\gamma} \tilde{N}^{\#} \Sigma J E^{\dagger} E J \Sigma \tilde{N}^{T} .
$$

Remark 2: For each fixed value of $\theta$, the problem is an LMI problem. Then, we can iterate on $\theta \in[0, \infty)$ and choose the value which minimizes the cost bound $(82)$ in Theorem 4

\section{ILLUSTRATIVE EXAMPLE}

In order to illustrate our methods and compare their performance, we use the same quantum system considered in [23] as an example. The system corresponds to a degenerate parametric amplifier and its $(S, L, H)$ description has the following form

$$
H=\frac{1}{2} i\left(\left(a^{\dagger}\right)^{2}-a^{2}\right), S=I, L=\sqrt{\kappa} a .
$$

We let the perturbation Hamiltonian be

$$
H_{2}=\frac{1}{2}\left[\begin{array}{ll}
a^{\dagger} & a^{T}
\end{array}\right]\left[\begin{array}{cc}
1 & 0.5 i \\
-0.5 i & 1
\end{array}\right]\left[\begin{array}{l}
a \\
a^{\#}
\end{array}\right]
$$

and the nominal Hamiltonian be

$$
H_{1}=\frac{1}{2}\left[\begin{array}{ll}
a^{\dagger} & a^{T}
\end{array}\right]\left[\begin{array}{cc}
-1 & 0.5 i \\
-0.5 i & -1
\end{array}\right]\left[\begin{array}{l}
a \\
a^{\#}
\end{array}\right]
$$

so that $H_{1}+H_{2}=H$. The corresponding parameters considered in Theorem 1, Theorem 2, Theorem 3 and Theorem 4 are as follows:

$$
\begin{aligned}
& M=\left[\begin{array}{cc}
-1 & 0.5 i \\
-0.5 i & -1
\end{array}\right], N=\left[\begin{array}{cc}
\sqrt{\kappa} & 0 \\
0 & \sqrt{\kappa}
\end{array}\right], \\
& F=\left[\begin{array}{cc}
-\frac{\kappa}{2}+i & 0.5 \\
0.5 & -\frac{\kappa}{2}-i
\end{array}\right], E=I
\end{aligned}
$$

and

$$
\Delta=\left[\begin{array}{cc}
1 & 0.5 i \\
-0.5 i & 1
\end{array}\right]
$$

To illustrate Theorem 1 and Theorem 3, we consider $H_{2} \in \mathcal{W}_{3}$. Hence, $\gamma=1$ is chosen to satisfy (22). The performance using the small gain approach for the uncertain quantum system is shown in Figure 1. In Figure 1, the dashed line represents the cost bound for the linear quantum system considered in Theorem 1 as a function of the parameter $\kappa$. The solid line shows the system performance with the coherent controller designed in Theorem 3. Compared to the performance without a controller, the coherent controller can guarantee that the system is stable for a larger range of the damping parameter $\kappa$ and gives the system improved performance.

Now we illustrate one approach to realizing the desired controller. For instance, when $\kappa=4.5$, by using the controller design method in Theorem 3, we have the desired controller Hamiltonian as

$$
H_{3}=\frac{1}{2}\left[\begin{array}{ll}
a^{\dagger} & a^{T}
\end{array}\right]\left[\begin{array}{cc}
0 & -0.5 i \\
0.5 i & 0
\end{array}\right]\left[\begin{array}{l}
a \\
a^{\#}
\end{array}\right] .
$$

This controller Hamiltonian can be realized by connecting the degenerate parametric amplifier with a static squeezer as shown in Figure 2. This static squeezer is a static Bogoliubov component which corresponds to the Bogoliubov transformation [27], [28]. Also, we have the following definition: 
Definition 1: (see [27], [28]) A static Bogoliubov component is a component that implements the Bogoliubov transformation: $\left[\begin{array}{c}d y(t) \\ d y^{\#}(t)\end{array}\right]=B\left[\begin{array}{c}d u(t) \\ d u^{\#}(t)\end{array}\right]$, where $B=$ $\left[\begin{array}{cc}B_{1} & B_{2} \\ B_{2}^{\#} & B_{1}^{\#}\end{array}\right], B^{\dagger} J B=J$.

To realize $H_{3}$ in 97 , we let the matrix $B=\left[\begin{array}{rr}\frac{5}{4} & -\frac{3}{4} \\ -\frac{3}{4} & \frac{5}{4}\end{array}\right]$ which satisfies the Bogoliubov condition $B^{\dagger} J B=J$, and $\tilde{\kappa}=\frac{1}{3}$. Therefore, the overall Hamiltonian of the closed loop system is $H=H_{1}+H_{2}+H_{3}$, which achieves the controller design goal. Detailed procedure regarding how to get the matrix $B$ can be found in appendix.

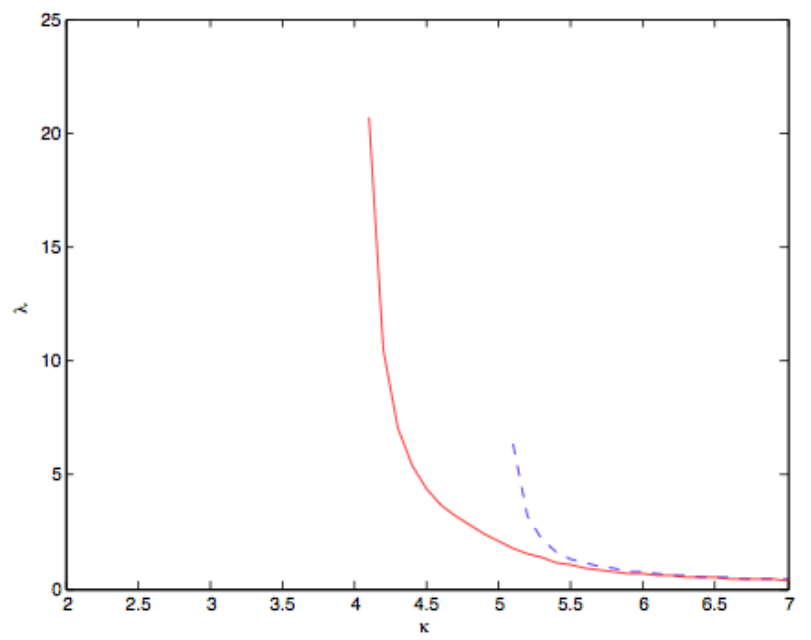

Fig. 1. Guaranteed cost bounds for an uncertain quantum system with a controller (solid line) and without a controller (dashed line) using the small gain approach

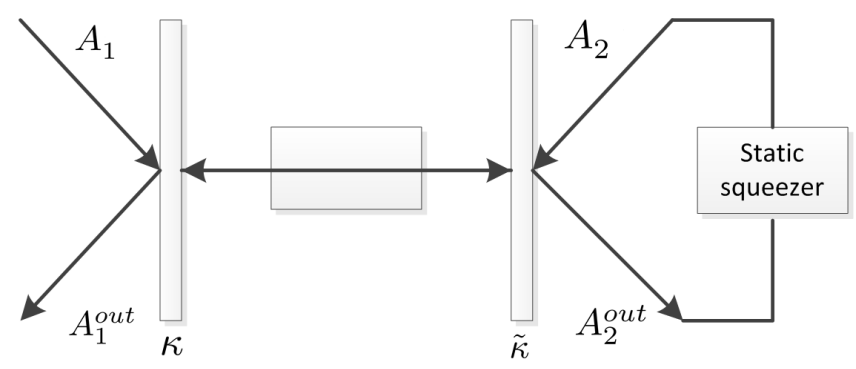

Fig. 2. Degenerate parametric amplifier coupled to a static squeezer.

To illustrate Theorem 2 and Theorem 4, we consider $H_{2} \in$ $\mathcal{W}_{4}$. Hence, $\gamma=2$ is chosen to satisfy (25). The results using the Popov approach are shown in Figure 3 and Figure 4. Figure 3 demonstrates how to choose the value of $\theta$. We consider the same example as above with $\kappa=3.8$ and iterate on $\theta \in[0,1]$. Figure 3 shows the cost bound for this quantum system obtained in Theorem 4 as a function of the parameter $\theta$. It is clear that the minimal cost bound is achieved when $\theta=0.1$. Therefore, we choose $\theta=0.1$ for $\kappa=3.8$ and use a similar method to choose $\theta$ for other values of $\kappa$.

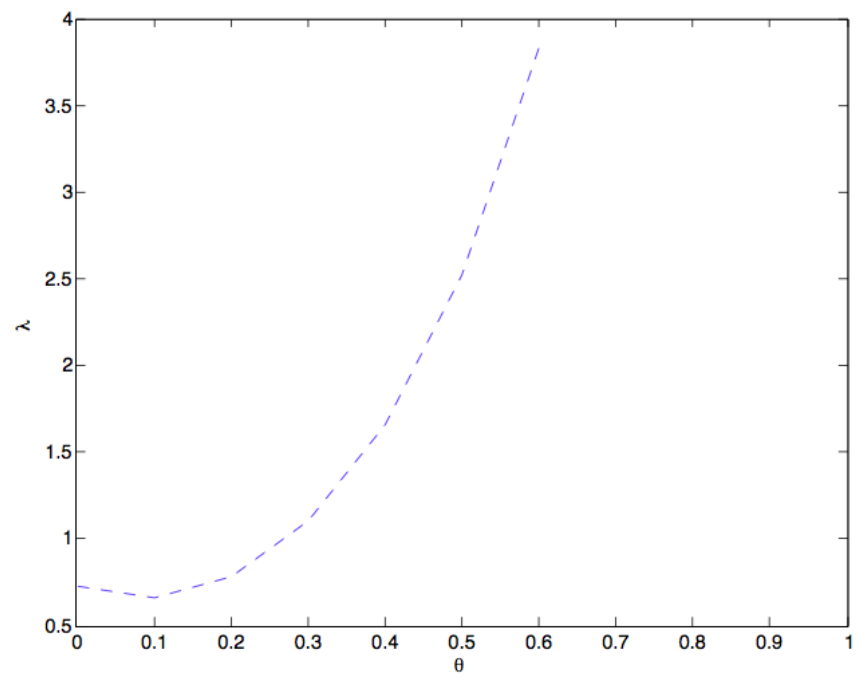

Fig. 3. Performance versus $\theta$

In Figure 4, the dashed line shows the performance for the given system considered in Theorem 2 and the solid line describes the cost bound for the linear quantum system with the coherent controller considered in Theorem 4. As can be seen in Figure 4, the system with a controller has better performance than the case without a controller.

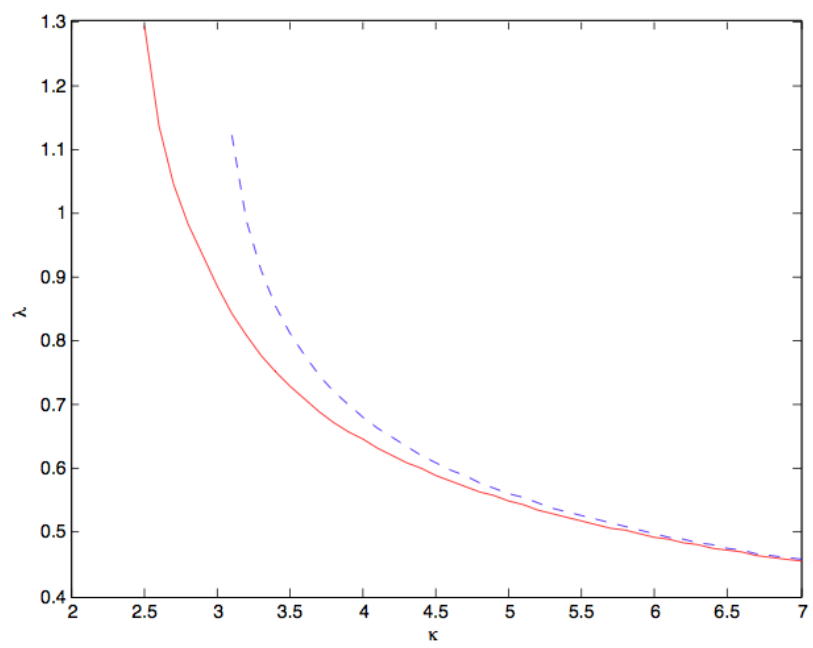

Fig. 4. Guaranteed cost bounds for the uncertain quantum system with a controller (solid line) and without a controller (dashed line) using the Popov approach

Also, we can observe that the method in Theorem 3 can only make the quantum system stable for $\kappa>4$ in the example. Therefore, compared with the results in Figure 1, the Popov method obtains a lower cost bound and a larger range of robust stability as shown in Figure 4. This is as expected, since the Popov approach allows for a more general class of Lyapunov functions than the small gain approach. 


\section{CONCLUSION}

In this paper, the small gain method and the Popov approach, respectively, are used to analyze the performance of an uncertain linear quantum system subject to a quadratic perturbation in the system Hamiltonian. Then, we add a coherent controller to make the given system not only stable but also to achieve improved performance. By an illustrative example, we have also shown that the Popov method adds to a considerable improvement over the small gain method in terms of system performance. Future work will include the extension of these approaches to nonlinear uncertain quantum systems [24].

\section{APPENDIX}

The detailed procedure regarding how to realize the desired controller is shown below. We consider a degenerate parametric amplifier (DPA) as an example. Based on the $(S, L, H)$ description in 92 , we can calculate the following quantum stochastic differential equations [1] describing the DPA:

$$
\begin{aligned}
{\left[\begin{array}{c}
d a(t) \\
d a^{\#}(t)
\end{array}\right]=} & {\left[\begin{array}{cc}
-\frac{\kappa}{2} & 1 \\
1 & -\frac{\kappa}{2}
\end{array}\right]\left[\begin{array}{c}
a(t) \\
a^{\#}(t)
\end{array}\right] d t } \\
& -\left[\begin{array}{cc}
\sqrt{\kappa} & 0 \\
0 & \sqrt{\kappa}
\end{array}\right]\left[\begin{array}{c}
d A_{1}(t) \\
d A_{1}^{\#}(t)
\end{array}\right] ; \\
d A_{1}^{\text {out }}(t)= & \sqrt{\kappa} a(t) d t+d A_{1}(t) .
\end{aligned}
$$

We have known that when $\kappa=4.5$ and using the controller design method in Theorem 3, we have the desired controller Hamiltonian as in 97.

Next, we show how to realize this controller Hamiltonian by connecting this degenerate parametric amplifier with a static squeezer as shown in Fig. 2. The corresponding quantum stochastic differential equations for this DPA is as follows:

$$
\begin{aligned}
{\left[\begin{array}{c}
d a(t) \\
d a^{\#}(t)
\end{array}\right]=} & {\left[\begin{array}{cc}
-\frac{\kappa+\tilde{\kappa}}{2} & 1 \\
1 & -\frac{\kappa+\tilde{\kappa}}{2}
\end{array}\right]\left[\begin{array}{c}
a(t) \\
a^{\#}(t)
\end{array}\right] d t } \\
- & {\left[\begin{array}{cc}
\sqrt{\kappa} & 0 \\
0 & \sqrt{\kappa}
\end{array}\right]\left[\begin{array}{c}
d A_{1}(t) \\
d A_{1}^{\#}(t)
\end{array}\right] } \\
& -\left[\begin{array}{cc}
\sqrt{\tilde{\kappa}} & 0 \\
0 & \sqrt{\tilde{\kappa}}
\end{array}\right]\left[\begin{array}{c}
d A_{2}(t) \\
d A_{2}^{\#}(t)
\end{array}\right] ; \\
d A_{1}^{\text {out }}(t)= & \sqrt{\kappa} a(t) d t+d A_{1}(t) \\
d A_{2}^{\text {out }}(t)= & \sqrt{\tilde{\kappa}} a(t) d t+d A_{2}(t) .
\end{aligned}
$$

We have known that this static squeezer is a static Bogoliubov component which satisfies Definition 1. According to the Definition 1, we have the following relation:

$$
\left[\begin{array}{c}
d A_{2}(t) \\
d A_{2}^{\#}(t)
\end{array}\right]=B\left[\begin{array}{c}
d A_{2}^{\text {out }}(t) \\
d A_{2}^{\text {out\# }}(t)
\end{array}\right]
$$

According to 98 , we have

$$
\begin{aligned}
{\left[\begin{array}{c}
d A_{2}^{\text {out }}(t) \\
d A_{2}^{\text {out } \#}(t)
\end{array}\right]=} & {\left[\begin{array}{cc}
\sqrt{\tilde{\kappa}} & 0 \\
0 & \sqrt{\tilde{\kappa}}
\end{array}\right]\left[\begin{array}{c}
a(t) \\
a^{\#}(t)
\end{array}\right] d t } \\
& +\left[\begin{array}{c}
d A_{2}(t) \\
d A_{2}^{\#}(t)
\end{array}\right]
\end{aligned}
$$

Substituting 99] into (100, we obtain that

$$
\begin{aligned}
B^{-1}\left[\begin{array}{l}
d A_{2}(t) \\
d A_{2}^{\#}(t)
\end{array}\right]= & {\left[\begin{array}{cc}
\sqrt{\tilde{\kappa}} & 0 \\
0 & \sqrt{\tilde{\kappa}}
\end{array}\right]\left[\begin{array}{c}
a(t) \\
a^{\#}(t)
\end{array}\right] d t } \\
& +\left[\begin{array}{c}
d A_{2}(t) \\
d A_{2}^{\#}(t)
\end{array}\right] .
\end{aligned}
$$

Hence,

$$
\left(B^{-1}-I\right)\left[\begin{array}{l}
d A_{2}(t) \\
d A_{2}^{\#}(t)
\end{array}\right]=\left[\begin{array}{cc}
\sqrt{\tilde{\kappa}} & 0 \\
0 & \sqrt{\tilde{\kappa}}
\end{array}\right]\left[\begin{array}{c}
a(t) \\
a^{\#}(t)
\end{array}\right] d t .
$$

We now assume inverse of $B^{-1}-I$ exists. It follows 101 that we can write

$$
\left[\begin{array}{l}
d A_{2}(t) \\
d A_{2}^{\#}(t)
\end{array}\right]=\left(B^{-1}-I\right)^{-1}\left[\begin{array}{cc}
\sqrt{\tilde{\kappa}} & 0 \\
0 & \sqrt{\tilde{\kappa}}
\end{array}\right]\left[\begin{array}{c}
a(t) \\
a^{\#}(t)
\end{array}\right] d t .
$$

Substituting (102) into first equation in 98 , we get

$$
\begin{aligned}
& {\left[\begin{array}{c}
d a(t) \\
d a^{\#}(t)
\end{array}\right]=\left[\begin{array}{cc}
-\frac{\kappa+\tilde{\kappa}}{2} & 1 \\
1 & -\frac{\kappa+\tilde{\kappa}}{2}
\end{array}\right]\left[\begin{array}{c}
a(t) \\
a^{\#}(t)
\end{array}\right] d t} \\
& -\left[\begin{array}{cc}
\sqrt{\kappa} & 0 \\
0 & \sqrt{\kappa}
\end{array}\right]\left[\begin{array}{l}
d A_{1}(t) \\
d A_{1}^{\#}(t)
\end{array}\right] \\
& -\left[\begin{array}{cc}
\sqrt{\tilde{\kappa}} & 0 \\
0 & \sqrt{\tilde{\kappa}}
\end{array}\right]\left(B^{-1}-I\right)^{-1} \\
& \times\left[\begin{array}{cc}
\sqrt{\tilde{\kappa}} & 0 \\
0 & \sqrt{\tilde{\kappa}}
\end{array}\right]\left[\begin{array}{c}
a(t) \\
a^{\#}(t)
\end{array}\right] d t \\
& =\left[\begin{array}{cc}
-\frac{\kappa+\tilde{\kappa}}{2} & 1 \\
1 & -\frac{\kappa+\tilde{\kappa}}{2}
\end{array}\right]\left[\begin{array}{c}
a(t) \\
a^{\#}(t)
\end{array}\right] d t \\
& -\left[\begin{array}{cc}
\sqrt{\kappa} & 0 \\
0 & \sqrt{\kappa}
\end{array}\right]\left[\begin{array}{l}
d A_{1}(t) \\
d A_{1}^{\#}(t)
\end{array}\right] \\
& -\left(B^{-1}-I\right)^{-1}\left[\begin{array}{cc}
\tilde{\kappa} & 0 \\
0 & \tilde{\kappa}
\end{array}\right]\left[\begin{array}{c}
a(t) \\
a^{\#}(t)
\end{array}\right] d t \text {. }
\end{aligned}
$$

According to Definition 1, the $B$ matrix satisfies the following relation:

$$
\begin{aligned}
J B^{\dagger} J B & =\left[\begin{array}{cc}
I & 0 \\
0 & -I
\end{array}\right]\left[\begin{array}{cc}
B_{1}^{\dagger} & B_{2}^{T} \\
B_{2}^{\dagger} & B_{1}^{T}
\end{array}\right]\left[\begin{array}{cc}
I & 0 \\
0 & -I
\end{array}\right]\left[\begin{array}{cc}
B_{1} & B_{2} \\
B_{2}^{\#} & B_{1}^{\#}
\end{array}\right] \\
& =\left[\begin{array}{cc}
B_{1}^{\dagger} & B_{2}^{T} \\
-B_{2}^{\dagger} & -B_{1}^{T}
\end{array}\right]\left[\begin{array}{cc}
B_{1} & B_{2} \\
-B_{2}^{\#} & -B_{1}^{\#}
\end{array}\right] \\
& =\left[\begin{array}{cc}
B_{1}^{\dagger} B_{1}-B_{2}^{T} B_{2}^{\#} & B_{1}^{\dagger} B_{2}-B_{2}^{T} B_{1}^{\#} \\
-B_{2}^{\dagger} B_{1}+B_{1}^{T} B_{2}^{\#} & -B_{2}^{\dagger} B_{2}+B_{1}^{T} B_{1}^{\#}
\end{array}\right]=I .
\end{aligned}
$$

In our case that $B$ is a $2 \times 2$ matrix, we have

$$
B_{1}^{\dagger} B_{2}-B_{2}^{T} B_{1}^{\#}=0 ;-B_{2}^{\dagger} B_{1}+B_{1}^{T} B_{2}^{\#}=0 .
$$

Moreover, we need to have the following relation:

$B_{1}^{\dagger} B_{1}-B_{2}^{T} B_{2}^{\#}=-B_{2}^{\dagger} B_{2}+B_{1}^{T} B_{1}^{\#}=\left(B_{1 x}^{2}+B_{1 y}^{2}\right)-\left(B_{2 x}^{2}+B_{2 y}^{2}\right)=I$.

where $B_{1}=B_{1 x}+i B_{1 y}, B_{2}=B_{2 x}+i B_{2 y}$. Thus, we can assume that

$$
B_{1 x}^{2}+B_{1 y}^{2}=\cosh (r)^{2} ; B_{2 x}^{2}+B_{2 y}^{2}=\sinh (r)^{2} .
$$

Hence, we may write the matrix $B$ in the following form:

$B=\left[\begin{array}{cc}\cosh (r) \cos (\alpha)+i \cosh (r) \sin (\alpha) & \sinh (r) \cos (\beta)+i \sinh (r) \sin (\beta) \\ \sinh (r) \cos (\beta)-i \sinh (r) \sin (\beta) & \cosh (r) \cos (\alpha)-i \cosh (r) \sin (\alpha)\end{array}\right]$. 
Since $B, I$ are $2 \times 2$ matrices, we have

$$
\left(B^{-1}-I\right)^{-1}=\frac{1}{2-2 B_{1 x}}\left[\begin{array}{cc}
B_{1}-1 & B_{2} \\
B_{2}^{\#} & B_{1}^{\#}-1
\end{array}\right] .
$$

Therefore, the last term on the right side of equation (103) can be expressed as:

$$
\begin{aligned}
& \tilde{\kappa}\left(B^{-1}-I\right)^{-1}=\frac{\tilde{\kappa}}{2-2 \cosh (r) \cos (\alpha)} \\
& \times\left(\left[\begin{array}{cc}
\cosh (r) \cos (\alpha)-1 & 0 \\
0 & \cosh (r) \cos (\alpha)-1
\end{array}\right]\right. \\
& \left.+\left[\begin{array}{cc}
\sinh (r) \cos (\beta)-i \sinh (r) \sin (\beta) & \sinh (r) \cos (\beta)+i \sinh (r) \sin (\beta)
\end{array}\right]\right) \\
= & {\left[\begin{array}{cc}
-\frac{\tilde{\kappa}}{2} & 0 \\
0 & -\frac{\tilde{\kappa}}{2}
\end{array}\right]+\frac{\tilde{\kappa} \cosh (r) \sin (\alpha)}{2-2 \cosh (r) \cos (\alpha)} } \\
& \times\left[\begin{array}{cc}
\sin (\alpha) & \sinh (r) \cos (\beta)+i \sinh (r) \sin (\beta) \\
\sinh (r) \cos (\beta)-i \sinh (r) \sin (\beta) & -i \cosh (r) \sin (\alpha)
\end{array}\right]
\end{aligned}
$$

Substituting (104) into (103), we have

$$
\begin{aligned}
& {\left[\begin{array}{c}
d a(t) \\
d a^{\#}(t)
\end{array}\right]=\left[\begin{array}{cc}
-\frac{\kappa}{2} & 1 \\
1 & -\frac{\kappa}{2}
\end{array}\right]\left[\begin{array}{c}
a(t) \\
a^{\#}(t)
\end{array}\right] d t} \\
& -\left[\begin{array}{cc}
\sqrt{\kappa} & 0 \\
0 & \sqrt{\kappa}
\end{array}\right]\left[\begin{array}{c}
d A_{1}(t) \\
d A_{1}^{\#}(t)
\end{array}\right] \\
& -\frac{\operatorname{\kappa }}{2-2 \cosh (r) \cos (\alpha)} \times \\
& {\left[\begin{array}{c}
\sinh (r) \cos (\beta)+i \sinh (r) \sin (\beta) \\
\sinh (r) \cos (\beta)-i \sinh (r) \sin (\beta)
\end{array}\right] d t .}
\end{aligned}
$$

Therefore, the closed loop system with DPA and a static squeezer has the dynamical equation (105). The difference between this closed loop system (10万) and the original uncertain quantum system (98) is the addition of the last term on right side of (105) which corresponds to the controller Hamiltonian $H_{3}$. To realize our desired controller Hamiltonian $H_{3}$ as in $[\overline{97]}$, the following relation is required:

$$
\begin{aligned}
& -\frac{\tilde{\kappa}}{2-2 \cosh (r) \cos (\alpha)} \times \\
& {\left[\begin{array}{c}
i \cosh (r) \sin (\alpha) \\
\sinh (r) \cos (\beta)-i \sinh (r) \sin (\beta)
\end{array}\right.} \\
& =-i J K=\left[\begin{array}{cc}
0 & -0.5 \\
-0.5 & 0
\end{array}\right] .
\end{aligned}
$$$$
\left.\begin{array}{c}
\sinh (r) \cos (\beta)+i \sinh (r) \sin (\beta) \\
-i \cosh (r) \sin (\alpha)
\end{array}\right]
$$

When $\alpha=0, \beta=0, \sinh (r)=-\frac{3}{4}, \cosh (r)=\frac{5}{4}, \tilde{\kappa}=\frac{1}{3}$, the relationship (106) holds. In this case, we may have $B$ matrix as $B=\left[\begin{array}{rr}\frac{5}{4} & -\frac{3}{4} \\ -\frac{3}{4} & \frac{5}{4}\end{array}\right]$ which satisfies the Bogoliubov condition $B^{\dagger} J B=J$.

\section{REFERENCES}

[1] M. R. James, H. I. Nurdin, and I. R. Petersen, " $H^{\infty}$ control of linear quantum stochastic systems," IEEE Transactions on Automatic Control, vol. 53, no. 8, pp. 1787-1803, 2008.

[2] I. R. Petersen, "Quantum linear systems theory," in Proceedings of the 19th International Symposium on Mathematical Theory of Networks and Systems, Budapest, Hungary, July 2010.

[3] D. Dong and I. R. Petersen, "Sliding mode control of quantum systems," New Journal of Physics, vol. 11, p. 105033, 2009.

[4] M. Yanagisawa and H. Kimura, "Transfer function approach to quantum control-part I: Dynamics of quantum feedback systems," IEEE Transactions on Automatic Control, vol. 48, no. 12, pp. 2107-2120, 2003.
[5] M. Yanagisawa and H. Kimura, "Transfer function approach to quantum control-part II: Control concepts and applications," IEEE Transactions on Automatic Control, vol. 48, no. 12, pp. 2121-2132, 2003.

[6] M. James and J. Gough, "Quantum dissipative systems and feedback control design by interconnection," IEEE Transactions on Automatic Control, vol. 55, no. 8, pp. 1806-1820, 2010.

[7] J. Gough and M. R. James, "The series product and its application to quantum feedforward and feedback networks," IEEE Transactions on Automatic Control, vol. 54, no. 11, pp. 2530-2544, 2009.

[8] I. R. Petersen, V. Ugrinovskii, and M. R. James, "Robust stability of uncertain quantum systems," in Proceedings of the 2012 American Control Conference, Montreal, Canada, June 2012.

[9] I. R. Petersen, V. Ugrinovskii, and M. R. James, "Robust stability of quantum systems with a nonlinear coupling operator," in Proceedings of the 51st IEEE Conference on Decision and Control, Maui, December 2012.

[10] I. R. Petersen, "Quantum robust stability of a small Josephson junction in a resonant cavity," in 2012 IEEE Multi-conference on Systems and Control, Dubrovnik, Croatia, October 2012.

[11] I. R. Petersen, "Robust stability analysis of an optical parametric amplifier quantum system," in Proceedings of the 2013 Asian Control Conference, Istanbul, Turkey, July 2013.

[12] I. R. Petersen, "Robust stability of quantum systems with nonlinear dynamic uncertainties," in Proceedings of the 52nd IEEE Conference on Decision and Control, Florence, Italy, December 2013.

[13] M. R. James, I. R. Petersen, and V. Ugrinovskii, "A Popov stability condition for uncertain linear quantum systems," in Proceedings of the 2013 American Control Conference, Washington, DC, USA, June 2013.

[14] I. R. Petersen, "Quantum Popov robust stability analysis of an optical cavity containing a saturated Kerr medium," in Proceedings of the 2013 European Control Conference, Zurich, Switzerland, July 2013.

[15] J. E. Gough, M. R. James, and H. I. Nurdin, "Squeezing components in linear quantum feedback networks," Physical Review A, vol. 81, p. 023804, 2010.

[16] H. M. Wiseman and G. J. Milburn, Quantum Measurement and Control, Cambridge, U.K.: Cambridge University Press, 2010.

[17] D. Dong and I. R. Petersen, "Quantum control theory and applications: a survey," IET Control Theory \& Applications, vol. 4, pp. 2651-2671, 2010.

[18] I. R. Petersen, V. Ugrinovskii, and M. R. James, "Robust stability of uncertain linear quantum systems," Philosophical Transactions of the Royal Society A, vol. 370, no. 1979, pp. 5354-5363, 2012.

[19] C. D'Helon and M. R. James. "Stability, gain, and robustness in quantum feedback networks," Physical Review A, vol. 73, p. 053803, 2006.

[20] H. I. Nurdin, M. R. James, and I. R. Petersen, "Coherent quantum LQG control," Automatica, vol. 45, no. 8, pp. 1837-1846, 2009.

[21] A. I. Maalouf and I. R. Petersen, "Coherent $H^{\infty}$ control for a class of linear complex quantum systems," IEEE Transactions on Automatic Control, vol. 56, no. 2, pp. 309-319, 2011.

[22] C. Xiang, I. R. Petersen and D. Dong, "Performance analysis and coherent guaranteed cost control for uncertain quantum systems," in the Proceedings of the 2014 European Control Conference, Strasbourg, France, June 2014

[23] C. Xiang, I. R. Petersen and D. Dong, "A Popov approach to performance analysis and coherent guaranteed cost control for uncertain quantum systems," in the Proceedings of the 2014 Australian Control Conference, Canberra, Australia, November 2014.

[24] I. R. Petersen, "Guaranteed non-quadratic performance for quantum systems with nonlinear uncertainties," in Proceedings of the 2014 American Control Conference, Portland, Oregon, USA, June 2014.

[25] H. Khalil, Nonlinear Systems, 3rd ed. Upper Saddle River, NJ, USA: Prentice-Hall, 2002.

[26] S. Boyd, L. El Ghaoui, E. Feron, and V. Balakrishnan, Linear Matrix Inequalities in Systems and Control Theory. Philadelphia, PA: SIAM, 1994.

[27] J. E. Gough, M. R. James, and H. I. Nurdin, "Squeezing components in linear quantum feedback networks," Physical Review A, vol. 81, p. 023804, 2010.

[28] S. L. Vuglar and I. R. Petersen, "Singular perturbation approximations for general linear quantum systems, in Proceedings of the Australian Control Conference, Sydney, Australia, Nov 2012, pp. 459463. 